Service social

\title{
Le cadre de travail et l'épuisement professionnel des intervenants sociaux en Gaspésie
}

\section{Jean-Pierre Villeneuve et André Beaudoin}

Volume 42, numéro 2, 1993

Recherche et pensée critique

URI : https://id.erudit.org/iderudit/706619ar

DOI : https://doi.org/10.7202/706619ar

Aller au sommaire du numéro

Éditeur(s)

École de service social de l'Université Laval

ISSN

1708-1734 (numérique)

Découvrir la revue

Citer cet article

Villeneuve, J.-P. \& Beaudoin, A. (1993). Le cadre de travail et l'épuisement professionnel des intervenants sociaux en Gaspésie. Service social, 42(2), 83-101. https://doi.org/10.7202/706619ar
Résumé de l'article

Cet article examine l'influence du cadre de travail sur l'épuisement professionnel d'un échantillon de 95 intervenants sociaux en Gaspésie. L'échelle d'environnement de travail (EET) de Moos et Insel et l'inventaire d'épuisement professionnel de Maslach sont utilisés pour mesurer les deux variables. L'absence de clarté dans la définition du cadre et des attentes de travail a une influence sur la fréquence et l'intensité de l'épuisement émotionnel des personnes en moins bonne santé et vivant en couple ; l'intensité de l'épuisement émotionnel est aussi plus forte pour les intervenants sans formation universitaire et sa fréquence est plus élevée lorsqu'ils font leur intervention en contexte d'autorité et dans un milieu où le nombre d'intervenants est plus élevé. Quant à l'absence de clarté, elle influence surtout la fréquence de l'épuisement émotionnel dans les milieux plus petits et en contexte d'autorité. 


\section{Le cadre de travail et l'épuisement professionnel des intervenants sociaux en Gaspésie ${ }^{1}$}

Jean-Pierre VILLENEUVE

Professeur

Cégep de la Gaspésie et des îles

André BEAUDOIN

Professeur

École de service social

Université Laval

Parler de l'épuisement professionnel, c'est aborder une réalité qui touche beaucoup d'employés dans le domaine des services sociaux. C'est un problème auquel plusieurs chercheurs se sont intéressés depuis un certain nombre d'années (Bernier, 1989a et b). Nous sommes tous témoins à un moment ou l'autre de notre vie professionnelle des ravages de ce mal insidieux qui n'affecte pas tout le monde, mais qui guette bon nombre $\mathrm{d}^{\prime}$ intervenants. C'est un sentiment d'impuissance face à cette réalité et le désir de comprendre davantage ses manifestations dans le milieu du travail qui sont à l'origine de la présente étude.

La recherche dont les résultats sont présentés ici a été conduite en 1990 et 1991 auprès d'intervenants dans le domaine des services sociaux en Gaspésie. Elle porte sur la relation entre l'environnement de travail et le phénomène de l'épuisement professionnel (burnout). Elle vise à répondre aux trois questions suivantes:

1. Le présent article part du mémoire de maîtrise de J.-P. Villeneuve (1991). 
- Quels sont les éléments du contexte de travail des intervenants qui sont source d'épuisement professionnel?

- Y a-t-il des différences selon les milieux de pratique?

- Les types, les corps d'emploi ou le nombre d'années d'expérience jouent-ils un rôle dans le développement du problème?

\section{L'ÉPUISEMENT PROFESSIONNEL ET L'ENVIRONNEMENT DE TRAVAIL}

L'état actuel des connaissances (Rosenthal et al.,1983; McCulloch and O'Brien,1986; Wade et al., 1986; Golembiewski et al.,1987; Fimian ,1984; Cherniss, 1980b) met en évidence l'influence d'un milieu de travail exigeant dans le développement du problème de l'épuisement professionnel. Pourtant, dans la pratique, comme le dit Diane Bernier (1989), le phénomène continue d'être traité surtout comme un problème d'attitude au travail. De façon générale, il y a peu d'interrogation sur le rôle que joue l'organisation elle-même en ce qui concerne l'épuisement de ses employés.

Par contre, l'unanimité est loin d'être faite autour des facteurs qui sont à l'origine du problème, comme on le verra plus loin. Toutefois, en ce qui concerne la définition du concept, malgré une certaine élasticité, les écarts sont moins marqués entre les auteurs. On attribue la paternité du concept au psychanalyste américain Freudenberger: il l'a utilisé pour la première fois en 1974 pour décrire l'état général de ceux qui s'étaient épuisés dans l'accomplissement d'une tâche (Freudenberger, 1982). Plusieurs équipes ont par la suite balisé le champ d'étude sur le phénomène: on retrouve entre autres Maslach et Jackson (1981), Pines, Aronson et Kafry (1982) ainsi que Cherniss (1980) pour ce qui est des services sociaux.

Dans l'ensemble des études, on s'entend pour dire que cet état d'épuisement est l'aboutissement d'un processus plus ou moins long qui peut mener graduellement l'individu vers une incapacité à accomplir ses tâches. Les études consultées adhèrent de façon majoritaire à la définition donnée par Maslach et Jackson (1981) qui le conçoivent comme :

un syndrome d'épuisement émotionnel et physique comprenant une image négative de soi-même, une attitude négative envers le travail, ainsi qu'une perte d'intérêt et de préoccupation envers la clientèle ${ }^{2}$ (Maslach et Jackson, traduit par Gevry et Alain, 1988: 61).

Cette définition sert ici de référence, car elle a l'avantage de bien coller au domaine des services sociaux et aux difficultés inhérentes à la relation d'aide. C'est elle qui a servi de référence dans cette étude.

2. Le caractère gras est des auteurs. 
Par ailleurs, dans l'exploration des facteurs reliés à l'épuisement professionnel, les auteurs recensés se placent sur un continuum où la personne est à une extrémité et où le cadre ou le milieu de travail est à l'autre. Leur position dépend de l'importance qu'ils accordent à ces deux catégories de facteurs. Pour Freudenberger (1982), c'est une question d'attitude de la personne, alors qu'à l'inverse pour Cherniss (1980b) c'est une réponse à une situation de travail intolérable.

Même si dans l'état actuel des connaissances, il paraît nécessaire de tenir compte d'un ensemble de facteurs sur le continuum personne-milieu de travail, aux fins de la présente recherche ce sont surtout les facteurs reliés à I'environnement de travail qui sont l'objet d'attention. Dans cette optique, Duquette, Kerouac et Beaudet (1990) font état d'une approche mieux intégrée élaborée par Courage et Williams (1987) au cours des dernières années. Celle-ci s'inspire de la théorie systémique:

Selon cette approche conceptuelle, l'épuisement professionnel résulte de I interdépendance de facteurs relatifs au personnel, à la clientèle et à l'organisation. Selon une optique circulaire, Courage et Williams (1987) rapportent que les variables initiatrices de l'épuisement professionnel ne sont pas isolées ou ne représentent pas une suite chronologique, successive et immuable d'événements, mais relèvent plutôt d'une interaction entre le personnel, l'organisation et la clientèle ${ }^{3}$ (p. 9).

Les études qui mettent en relation les facteurs associés à l'environnement de travail et l'épuisement dégagent un certain nombre d'éléments pour circonscrire cet environnement ou ce cadre de travail. Fimian (1984), McCullogh et O'Brien (1986), de même que Cherniss (1980b), élaborent des conceptions du cadre de travail qui présentent des points communs en ce qui concerne la structure, l'exercice du pouvoir, l'accomplissement des rôles, les relations et la satisfaction des besoins.

Néanmoins, quatre études qui traitent plus spécialement de l'environnement de travail ( Wade et al., 1986; Rosenthal et al., 1983; Golembiewski et al., 1987; Constable et Russel, 1986) ont fait référence à Moos et Insel (1974) pour définir cet environnement et ont utilisé le même instrument pour l'évaluer: la "work environment scale» (WES) ou en français échelle d'environnement du travail (EET).

La notion de cadre de travail définie par Moos et Insel comporte trois grandes dimensions: le «design» organisationnel, les relations entre les individus et la possibilité de croissance et de développement offerte par le milieu. Ces trois dimensions réunissent l'ensemble des éléments qui constituent

3. Le caractère gras est des auteurs. 
le climat social d'un milieu de travail et ont un impact sur le degré de stress que vivent les employés.

1. Les relations entre les personnes ou «interrelations » font référence à l'engagement personnel, à la cohésion entre les employés et à celle qu'on retrouve dans la supervision et le soutien professionnel.

2. La possibilité de croissance personnelle se fonde sur la pression provenant du travail, l'incitation à l'autonomie et la nature de la tâche.

3. Le «design» organisationnel caractérise le maintien et les possibilités de changement du système. II concerne la clarté dans la définition des tâches et dans l'exercice du contrôle; il est relié au sentiment de confort physique dans l'organisation et à la possibilité d'innover.

Cette conception met en lumière un large éventail d'éléments constituant l'environnement de travail qui sont retenus dans notre analyse. En même temps, on tiendra aussi compte d'un certain nombre de caractéristiques personnelles des répondants dont il sera question plus loin.

Comme on peut le voir dans le schéma de la figure 1, la présente recherche vise surtout à établir les relations entre le cadre de travail tel qu'il est vécu par les intervenants et leur épuisement professionnel. En même temps, certaines variables qui sont des caractéristiques individuelles ou reliées à la position de l'employé dans l'établissement sont utilisées comme variables de contrôle.

FigURE 1

Cadre conceptuel

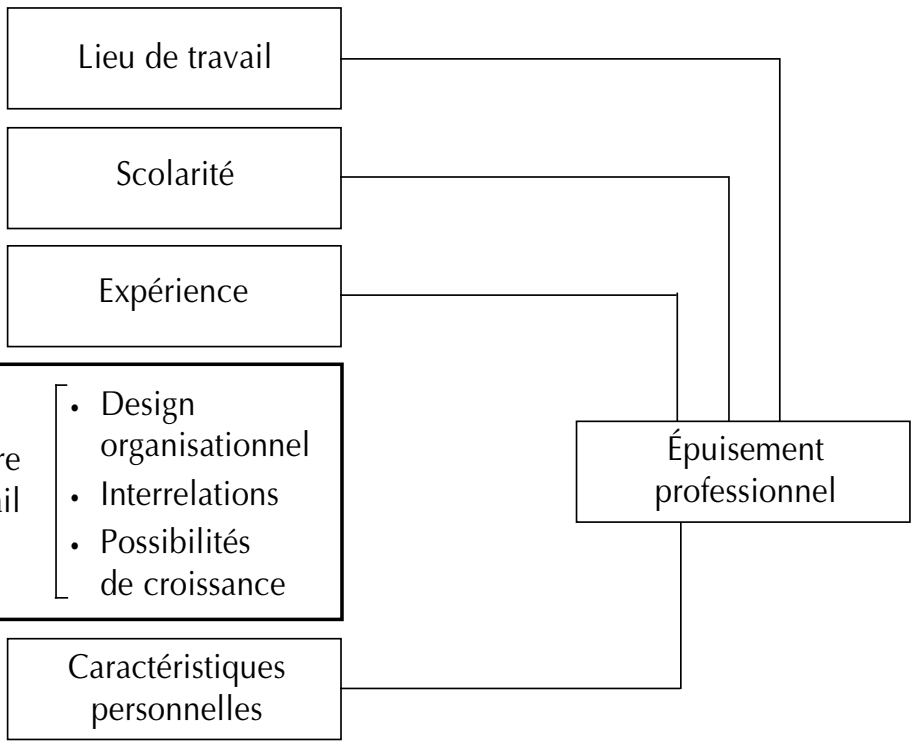

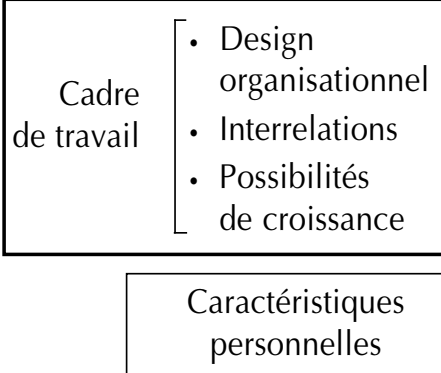




\section{MÉTHODE UTILISÉE}

La population de l'étude est composée de 126 intervenants psychosociaux (techniciens en assistance sociale [TAS], travailleurs sociaux [TS] et agents de relations humaines $[\mathrm{ARH}])$ travaillant dans six succursales du Centre de services sociaux (CSS) ${ }^{4}$ et six centres locaux de services communautaires (CLSC) répartis sur le territoire gaspésien (incluant les îles-de-la-Madeleine).

Le questionnaire a été adressé à chaque intervenant en mai 1990. Il était accompagné d'une lettre avec des directives précises et une enveloppe de retour affranchie et adressée au domicile du responsable de l'enquête. Le taux de réponses a été très satisfaisant, 95 questionnaires (76\%) ayant été remplis et retournés: $90 \%$ des répondants provenaient du CSS et $60 \%$ des CLSC.

Le questionnaire de 128 questions comprend trois sections: la première contient l'échelle d'environnement de travail (90 questions), la seconde l'échelle d'épuisement professionnel de Maslach (22 questions) et la troisième concerne les caractéristiques socio-démographiques utilisées comme variables de contrôle (16 questions).

\section{PORTRAIT DES RÉPONDANTS ET DU CONTEXTE DE TRAVAIL}

Les répondants sont à $63 \%$ des intervenantes. Ils sont âgés en moyenne de 33,4 ans (écart type de 7,3 ) et la médiane se situe à 32 ans. Les deux tiers vivent en couple. Ils ont en majorité (72\%) une formation universitaire de baccalauréat, surtout en travail social, les autres ayant une formation collégiale ou un certificat. Le portrait des caractéristiques de leur milieu de travail et de leur expérience professionnelle est le suivant:

- Endroit de travail: 56 \% travaillent en CSS; les autres en CLSC;

- Statut et type d'emploi: plus des quatre cinquièmes sont permanents et seulement 12,6\% ne sont pas à plein temps ;

- Taille des lieux de travail: selon le nombre d'intervenants dans les lieux de travail, les répondants se répartissent ainsi :

a) seulement 6 travaillent dans des points de service avec 3 à 5 intervenants ;

b) les points de service où sont $31,6 \%$ des répondants comptent 8 à 10 praticiens sociaux;

c) $44 \%$ des répondants travaillent dans des endroits où il y a 11 ou 12 intervenants;

4. L'étude a été menée avant la réforme d'avril 1993. C'est pourquoi le terme CSS est utilisé ici. 
d) le lieu de travail de $18 \%$ des participants à l'étude compte 15 ou 16 praticiens.

- Domaine de pratique: les répondants, à 35,8\% (34), exercent leur tâche dans un contexte d'autorité (protection de la jeunesse, jeunes contrevenants) ; 44,2\% (42) travaillent en contexte volontaire (adultes, personnes âgées, adoption, planification des naissances ou intervenant en scolaire en CLSC); quant au reste, un nombre plus restreint de 11 employés $(11,6 \%$ ) sont affectés au maintien à domicile en CLSC ou au secteur ressource en CSS et seulement huit répondants travaillent comme organisateurs communautaires;

- Expérience professionnelle: de ce côté il y a une représentation plus forte des répondants avec moins d'expérience professionnelle: I'écart type (6,9 ans) est presque aussi grand que la moyenne (8,9 ans). Cette tendance est encore plus accentuée pour ce qui est de la durée de l'expérience du séjour dans l'établissement: plus de $53 \%$ des répondants ont moins de 5 ans d'expérience dans l'établissement et l'écart type est, cette fois, plus grand que la moyenne $(6,4$ vs 6,3 ans).

\section{LA RÉPARTITION DES RÉPONDANTS SELON LEUR PERCEPTION DU CADRE DE TRAVAIL}

Le tableau 1 fait la représentation schématique des dix sous-échelles de l'échelle de l'environnement de travail (EET) développée par Moos et Insel (1974) et utilisée ici pour mesurer les trois grandes dimensions de l'environnement de travail : « interrelations », croissance personnelle et « design » organisationnel.

TABLEAU 1

Répartition des sous-échelles de l'EET selon la dimension

\begin{tabular}{|c|c|c|c|}
\hline Dimension : & « Interrelations » & $\begin{array}{l}\text { Possibilités } \\
\text { de croissance }\end{array}$ & $\begin{array}{l}\text { "Design » } \\
\text { organisationnel }\end{array}$ \\
\hline \multirow[t]{4}{*}{ Sous-échelles } & $\begin{array}{l}\text { Implication } \\
\text { (imp.) }\end{array}$ & $\begin{array}{l}\text { Autonomie } \\
\text { (aut.) }\end{array}$ & $\begin{array}{l}\text { Clarté } \\
\text { (clar.) }\end{array}$ \\
\hline & $\begin{array}{l}\text { Cohésion/pers. } \\
\text { (c.p.) }\end{array}$ & $\begin{array}{l}\text { Orientation } \\
\text { de la tâche (o.t.) }\end{array}$ & $\begin{array}{l}\text { Contrôle } \\
\text { (cont.) }\end{array}$ \\
\hline & $\begin{array}{l}\text { Soutien prof. } \\
\text { (s.p.) }\end{array}$ & $\begin{array}{l}\text { Pression } \\
\text { du travail (p.t.) }\end{array}$ & $\begin{array}{l}\text { Innovation } \\
\text { (inn.) }\end{array}$ \\
\hline & & & $\begin{array}{l}\text { Confort physique } \\
\text { (conf.) }\end{array}$ \\
\hline
\end{tabular}


L'instrument de Moos et Insel fournit dix scores, un pour chaque souséchelle. Il est possible de comparer chacun de ces résultats à une moyenne établie par les auteurs. Celle-ci a été déterminée à partir des résultats au test d'une population considérable, soit plus de 1442 personnes. Cette dernière constitue leur échantillon de référence (normative samples). Ainsi, un milieu peut être au-dessus ou au-dessous de la moyenne lorsqu'on compare chacun de ces résultats au résultat moyen qui est de 50 sur la courbe. La ligne centrale de la figure 2 indique ce score moyen de Moos et Insel.

FigURE 2

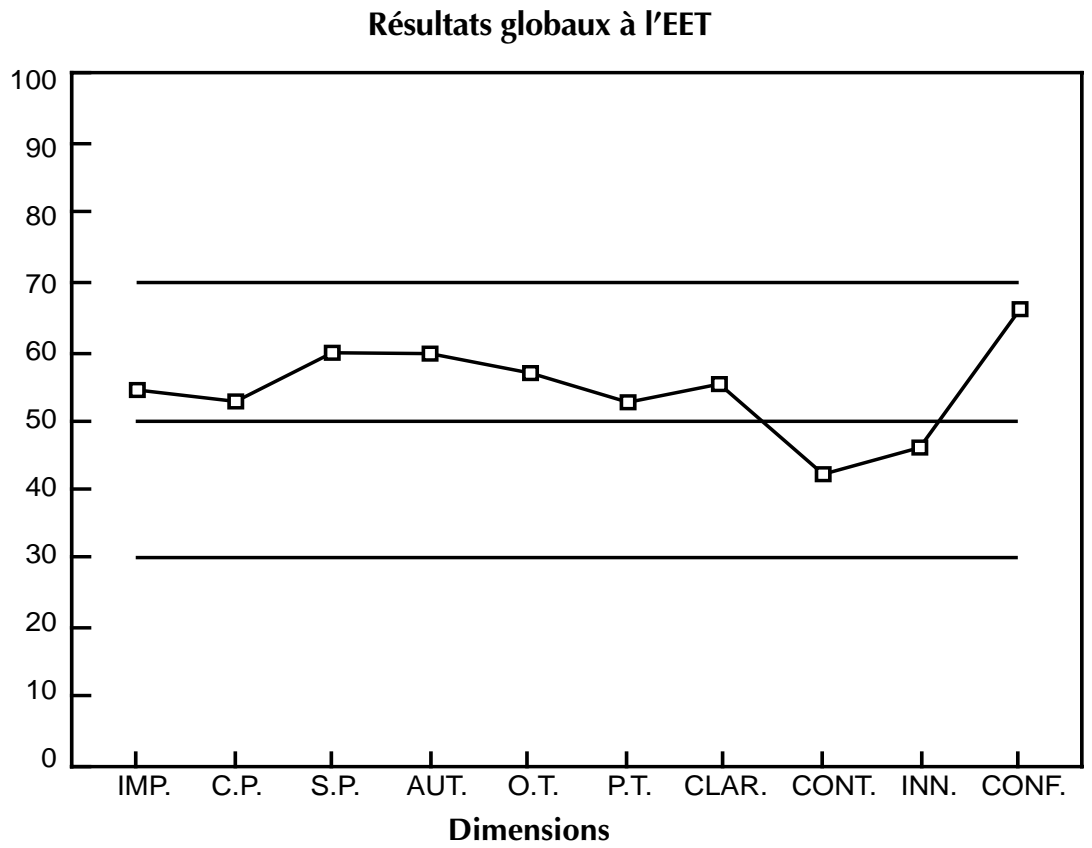

Lorsqu'on fait l'analyse des résultats des trois sous-échelles de la dimension «interrelations», on voit que les intervenants viennent de milieux où l'accent est mis sur les relations entre les personnes. Les répondants ont des scores qui se situent au-dessus de la moyenne pour les trois premières souséchelles. Comme l'indiquent les résultats de la première, l'échelle d'implication (imp.), les employés perçoivent dans leur milieu des possibilités d'entraide mutuelle plus forte que la moyenne. Leur perception du degré de cohésion dans l'administration du personnel est aussi plus élevée que la moyenne comme l'indique la position des répondants sur l'échelle correspondante (c.p.). Enfin, les résultats sur l'échelle de soutien professionnel (s.p.) indiquent un degré plus considérable de soutien et d'encouragement reçu par les travailleurs. 
Quant aux possibilités de croissance personnelle mesurées par l'échelle d'autonomie, celle d'orientation de la tâche et celle de la pression du travail, les résultats sont eux aussi au-dessus de la moyenne. C'est donc dire que les employés se sentent encouragés à être autonomes et à prendre des décisions par eux-mêmes (aut.) ; ils estiment aussi qu'une importance est accordée à la bonne planification, à l'efficacité et à l'exécution effective des tâches (o.t.) ; ils acceptent la pression existante dans le milieu concernant la charge (quantité) de travail et le temps accordé pour le faire (s.p.).

Pour ce qui est de la dernière dimension, celle du «design » organisationnel qui comporte quatre sous-échelles, les résultats sont plus partagés. En effet, pour ce qui est des deux sous-échelles de contrôle (cont.) et d'innovation (inn.), le milieu de travail est perçu par les répondants comme un contexte où le contrôle et l'innovation sont en deçà de la moyenne. C'est donc dire qu'on y trouve la perception d'une moins grande articulation dans la façon dont les supérieurs immédiats utilisent les règlements pour mettre de la pression sur les employés et les garder sous leur contrôle (cont.); en même temps, on perçoit une importance moindre accordée à la variété, au changement et aux nouvelles approches dans les façons d'accomplir le travail (inn.). Par contre, les employés expriment un degré de connaissance plus élevé des attentes reliées à leur routine quotidienne et de la clarté avec laquelle sont communiquées les règles et les politiques les concernant (clar.); ils occupent aussi une position plus élevée sur l'échelle de confort physique (conf.): la manière avec laquelle l'environnement physique contribue à créer un milieu de travail agréable pour eux est nettement plus élevée que ce qui a été constaté en moyenne par Moos et Insel.

\section{LE DEGRÉ D'ÉPUISEMENT PROFESSIONNEL DES INTERVENANTS}

À partir de la définition donnée précédemment de l'épuisement professionnel, l'échelle de Maslach est conçue pour évaluer les trois aspects du syndrome d'épuisement professionnel : l'épuisement émotionnel (EE), c'està-dire le sentiment d'être trop sollicité et vidé émotionnellement par son travail ; I'absence d'accomplissement personnel (AP) qui renvoie à l'absence de sentiment de compétence et de réalisation professionnelle dans le travail avec les clients; et la dépersonnalisation (DP), qui est le degré de réponse impersonnelle et dénuée de sentiment aux demandes du service, des clients ou de l'administration.

Chaque aspect est mesuré séparément par deux sous-échelles, une pour la fréquence du sentiment $(\mathrm{F})$ et l'autre pour son intensité (I). Ainsi on obtient à l'échelle de Maslach six scores qui correspondent à six aspects à partir des trois dimensions du syndrome de l'épuisement mentionnées plus haut. II est 
possible de qualifier ces résultats en se référant à des catégories élaborées par Maslach. Le degré d'épuisement dans chaque sous-échelle peut ainsi être faible, modéré ou élevé. Les résultats observés pour les intervenants (voir figure 3) sont les suivants:

- La fréquence de la dépersonnalisation (DP-F) est en moyenne à 5,45 sur l'échelle (écart type 4,26), ce qui la situe à la limite inférieure de modéré (entre 5 et 11 selon Maslach); son intensité (DP-I), avec une moyenne de 8,36 et un écart type de 5,90, est elle aussi modérée, puisqu'elle se situe entre 7 et 14 sur l'échelle de Maslach.

- Pour ce qui est de l'absence d'accomplissement personnel, sa fréquence (AP-F) a une moyenne de 35,75 et un écart type de 7, ce qui fait partie de la catégorie des modérés (entre 34 et 39$)^{5}$; il en est de même pour son intensité (AP-I) avec une moyenne de 38,89 et un écart type de 9,46, comparativement à un épuisement modéré qui se situe entre 37 et 43 selon Maslach.

Figure 3

Résultats globaux à l'échelle de Maslach

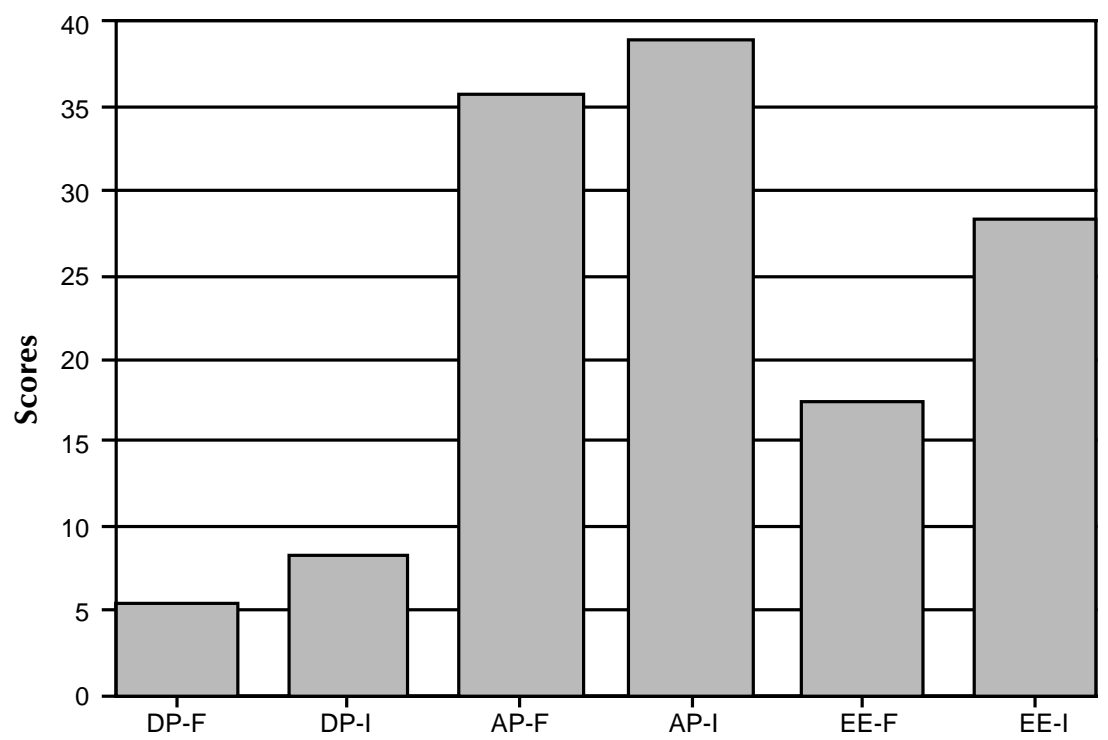

Dimensions

5. Soulignons que l'échelle d'accomplissement personnel est une échelle inversée. Plus le score est faible, plus le sentiment d'absence d'accomplissement personnel est élevé et, inversement, plus le score est élevé, plus le sentiment d'absence d'accomplissement personnel est faible. 
- Quant à l'épuisement émotionnel, la moyenne se situe à 17,73 avec un écart type de 9,46 sur l'échelle de la fréquence (EE-F), ce qui est à la limite inférieure d'un épuisement modéré (entre 18 et 29); sur l'échelle d'intensité (EE-I), avec une moyenne de 28,57 et un écart type de 12,58, on est aussi en présence d'intervenants ayant un épuisement émotionnel modéré (entre 26 et 39 selon Maslach). Ils vivent donc assez fréquemment et assez intensément les trois sentiments que sont la dépersonnalisation, l'absence d'accomplissement personnel et l'épuisement émotionnel.

\section{INFLUENCE DU CADRE DE TRAVAIL SUR L'ÉPUISEMENT}

L'analyse et l'interprétation des résultats des relations entre le cadre de travail et l'épuisement professionnel ont d'abord été effectuées avant d'examiner les caractéristiques personnelles.

\section{Les relations entre le cadre de travail et l'épuisement}

Jusqu'à quel point les différents éléments du cadre de travail dans lequel se retrouvent les intervenants sont-ils en relation avec leur degré plus ou moins élevé d'épuisement professionnel ? La réponse à cette question est fournie par I'analyse des coefficients de corrélation entre les dix sous-échelles du EET et les six sous-échelles du test de Maslach (voir tableau 2).

TABLEAU 2

Relation entre les variables du cadre de travail et les sous-échelles du test de Maslach

\begin{tabular}{lrrrrrr}
\hline $\begin{array}{l}\text { Test de } \\
\text { Maslach / EET }\end{array}$ & DP-I & DP-F & AP-I & AP-F & EE-I & EE-F \\
\hline Implication & $-0,24^{*}$ & $-0,14$ & 0,09 & $0,26^{*}$ & $-0,15$ & $-0,19$ \\
Cohésion & $-0,14$ & $-0,02$ & 0,04 & 0,12 & $-0,09$ & $-0,13$ \\
Soutien & & & & & & \\
$\quad$ professionnel & $-0,16$ & $-0,06$ & 0,12 & $0,24^{*}$ & $-0,04$ & $-0,08$ \\
Autonomie & $-0,16$ & $-0,02$ & 0,05 & $0,21^{*}$ & 0,11 & 0,05 \\
Orient. de tâche & $-0,10$ & 0,04 & 0,13 & 0,15 & 0,01 & $-0,03$ \\
Pression du travail & 0,17 & 0,18 & 0,04 & $-0,05$ & $0,40^{*}$ & $0,52^{*}$ \\
Clarté & $-0,20^{*}$ & 0,03 & 0,05 & $0,23^{*}$ & $-0,21^{*}$ & $-0,52^{*}$ \\
Contrôle & 0,11 & 0,17 & $-0,25^{*}$ & $-0,15$ & $-0,07$ & $-0,01$ \\
Innovation & $-0,03$ & $-0,00$ & 0,16 & 0,19 & $-0,02$ & $-0,12$ \\
Confort physique & $-0,09$ & $-0,08$ & $-0,10$ & 0,06 & $-0,05$ & $-0,15$ \\
\hline
\end{tabular}

$* p \leq 0,05$ 
À partir des onze coefficients de corrélation significatifs observés entre le cadre de travail et l'épuisement professionnel, on peut faire les constatations suivantes:

- La clarté est l'aspect de l'environnement de travail qui est le plus souvent relié significativement à l'épuisement émotionnel (EE-F) $(r=-$ 0,52): moins les patrons ont des attentes claires concernant la tâche et la routine quotidienne de leurs subalternes, plus ces derniers sont susceptibles d'avoir un épuisement émotionnel élevé. Par ailleurs, même si la relation est plus faible, elle n'en continue pas moins de demeurer significative $(p<0,05)$ entre la clarté du travail et les trois sous-échelles d'intensité de l'épuisement professionnel (DP-I, AP-I et EE-I). C'est donc dire que moins la tâche est clairement définie, plus on est susceptible d'être en présence d'un degré élevé d'intensité d'épuisement émotionnel et de dépersonnalisation et en même temps d'une insatisfaction plus élevée dans le degré d'accomplissement des tâches de la part des employés.

- La pression du travail est, quant à elle, assez fortement associée à la fréquence de l'épuisement émotionnel (EE-F) et à un degré moindre à son intensité (EEI). Plus la pression du travail est forte sur les employés, plus ils sont susceptibles d'avoir le sentiment d'être trop fréquemment et trop intensément sollicités émotionnellement dans leur tâche de travail $(r=0,52$ et $r=0,40)$.

- Quant aux autres dimensions du cadre de travail, lorsqu'elles sont en relations significatives, $c^{\prime}$ est à un degré moindre qu'elles sont reliées à certains aspects de l'épuisement professionnel.

Par ailleurs, aucune variable du cadre de travail n'est suffisante pour prédire ( $p>0,05)$ l'épuisement professionnel des intervenants de l'étude selon I'une ou l'autre des six échelles utilisées ici (voir tableau 3).

TABLEAU 3

Régressions multiples des aspects du cadre reliés aux dimensions de l'épuisement

\begin{tabular}{lcc}
\hline Éléments du cadre de travail & $\begin{array}{l}\text { Coefficient } \\
\text { de dét. mult. }\end{array}$ & $\begin{array}{c}\text { Dimensions } \\
\text { de l'épuisement }\end{array}$ \\
\hline $\begin{array}{l}\text { Implication (-) Clarté (-) } \\
\text { (Aucun élément) }\end{array}$ & 0,0593 & DP-I \\
Contrôle (-) & & AP-I \\
$\begin{array}{l}\text { Implication (+) Autonomie (+) } \\
\text { Soutien prof. (+) Clarté (+) }\end{array}$ & 0,0673 & AP-F \\
$\begin{array}{l}\text { Pression du travail (+) } \\
\text { Clarté (-) }\end{array}$ & 0,1572 & EE-I \\
$\begin{array}{l}\text { Pression du travail (+) } \\
\text { Clarté (-) }\end{array}$ & 0,2655 & EE-F \\
\hline
\end{tabular}




\section{Caractéristiques personnelles et épuisement}

En plus des constatations précédentes, il faut se demander s'il existe un lien entre les caractéristiques personnelles des répondants et l'épuisement professionnel. C'est pourquoi les relations entre certaines caractéristiques personnelles et l'épuisement professionnel ont été explorées. Cet exercice a permis de relever un certain nombre de caractéristiques personnelles qui ont une relation significative avec la variable dépendante.

Pour les variables personnelles mesurables selon une échelle ordinale ou d'intervalle, des analyses de corrélation ont été réalisées comme précédemment. Pour les autres, des analyses de khi carré ont servi à examiner le lien avec les souséchelles d'épuisement; à cet effet, les sous-échelles de Maslach ont été regroupées en trois catégories selon les indications de cette dernière. La synthèse des résultats de ces analyses faites aux tableaux 4 et 5 permet de dégager les constatations suivantes concernant chacune des sous-dimensions de l'épuisement professionnel:

1. Les intervenantes font plus souvent et plus intensivement face que les intervenants à l'épuisement émotionnel (EE-I et EE-F). II n'y a par ailleurs aucune différence entre les deux pour les autres sous-dimensions.

2. Les mêmes constatations s'appliquent à la situation de vie: les gens seuls comparés à ceux qui vivent en couple ont un épuisement émotionnel (EE-I et EE-F) plus élevé.

3. Les répondants avec une formation universitaire expriment une moins grande intensité de dépersonnalisation (DP-I) que ceux qui ont une formation non universitaire. De la même manière, ceux qui ont une formation complémentaire (formation en cours d'emploi) ont une moins forte intensité d'épuisement émotionnel (EE-I) que ceux qui n'en ont pas.

4. La fréquence de l'épuisement émotionnel (EE-F) est plus forte dans les points de service qui comptent dix intervenants ou plus que dans ceux où le nombre est plus restreint. Par ailleurs, la dépersonnalisation est ressentie de façon plus intensive en CSS qu'en CLSC.

5. Quant au domaine de pratique, ce sont les intervenants en organisation communautaire qui vivent le moins souvent de l'épuisement émotionnel (EE-F).

6. Les intervenants qui qualifient leur état de santé de passable ont plus souvent tendance à l'épuisement professionnel aussi bien dans les sousdimensions de dépersonnalisation (DP-I et DP-F) que dans celles d'épuisement émotionnel (EE-I et EE-F).

7. Même s'il s'agit d'une relation plutôt faible, on constate que plus le nombre d'années d'expérience dans le poste et l'établissement augmente, moins le sentiment qu'ont les employés de se réaliser dans leur travail est fort (AP-I). 
8. Quant aux autres variables, qu'il s'agisse de l'âge, de l'expérience professionnelle, du statut d'emploi, du type d'emploi ou de la présence de dépression dans la famille, aucune d'entre elles n'a une relation significative avec les différentes dimensions de l'épuisement professionnel.

\section{TABLeau 4}

Valeur du khi carré de la relation entre les variables personnelles nominales ou ordinales et les sous-échelles du test de Maslach

\begin{tabular}{lrrrrrr}
\hline $\begin{array}{l}\text { Test de Maslach/ } \\
\text { variables pers. }\end{array}$ & DP-I & DP-F & $\boldsymbol{A P}$-I & $\boldsymbol{A P}-\boldsymbol{F}$ & $\boldsymbol{E E}-\boldsymbol{I}$ & $\boldsymbol{E E}-\boldsymbol{F}$ \\
\hline Sexe $^{1}$ & 0,967 & 3,317 & 2,689 & 0,244 & $8,762^{*}$ & $17,290^{* *}$ \\
Situation de vie $^{1}$ & 0,127 & 0,886 & 2,403 & 2,530 & $8,811^{*}$ & $10,638^{*}$ \\
Formation $^{1}$ & $6,611^{*}$ & 4,403 & 1,434 & 3,472 & 0,026 & 2,261 \\
Autre formation $^{1}$ & 2,539 & 0,364 & 0,336 & 0,135 & $7,906^{*}$ & 4,369 \\
Nombre d'inter. $^{3}$ & 6,540 & 6,345 & 4,435 & 5,215 & 12,212 & $13,604^{*}$ \\
Domaine prat. $^{3}$ & 11,270 & 10,819 & 4,398 & 11,036 & 7,816 & $13,407^{*}$ \\
Statut d'emploi $^{1}$ & 1,279 & 1,306 & 5,553 & 5,366 & 5,142 & 1,453 \\
Type d'emploi $^{1}$ & 2,391 & 0,025 & 1,947 & 0,527 & 0,755 & 0,381 \\
Type d'établ. $^{1}$ & $6,396^{*}$ & 0,938 & 0,249 & 1,402 & 1,743 & 2,242 \\
Santé $^{2}$ & $17,73^{* *}$ & $21,485^{* *}$ & 5,184 & 5,186 & $11,988^{*}$ & $21,239^{* *}$ \\
Dépres. fam. $^{1}$ & 2,491 & 1,294 & 0,395 & 2,160 & 5,288 & 0,486 \\
& & & & & & \\
\hline
\end{tabular}

$* p \leq 0,05 * * p \leq 0,001 \quad$ 1. Degré de liberté $=2 \quad 2$. Degré de liberté $=4 \quad 3$. Degré de liberté $=6$

\section{TABLEAU 5}

Corrélation entre les variables personnelles métriques et les sous-échelles du test de Maslach

\begin{tabular}{lrrrrrr}
\hline $\begin{array}{l}\text { Test de Maslach/ } \\
\text { variables pers. }\end{array}$ & DP-I & DP-F & $\boldsymbol{A P}-\boldsymbol{I}$ & $\boldsymbol{A P}-\boldsymbol{F}$ & $\boldsymbol{E E}-\boldsymbol{I}$ & $\boldsymbol{E E}-\boldsymbol{F}$ \\
\hline Âge & 0,067 & 0,119 & $-0,198$ & 0,067 & 0,060 & 0,078 \\
Expér. poste & 0,056 & $-0,000$ & $-0,200^{*}$ & $-0,045$ & 0,048 & $-0,084$ \\
Expér. établ. & 0,100 & 0,074 & $-0,202^{*}$ & 0,099 & 0,033 & $-0,041$ \\
Expér. prof. & 0,019 & 0,094 & $-0,191$ & 0,080 & 0,013 & 0,027
\end{tabular}




\section{L'influence de la pression du travail et de la clarté sur l'épuisement émotionnel à la lumière des variables personnelles}

Dans une troisième étape de l'analyse, il s'agit de vérifier l'existence d'un rapport entre le cadre de travail et l'épuisement professionnel lorsque les caractéristiques personnelles des répondants sont introduites comme variables de contrôle dans la relation. Plus précisément, nous examinerons deux séries de relations particulières pour lesquelles les coefficients de détermination constatés dans le tableau 3 sont plus élevés: celles de l'influence de la pression du travail et de la clarté de la tâche sur l'épuisement émotionnel.

Comme nous l'avons constaté précédemment, plus la pression du travail est forte, plus l'intensité et la fréquence de l'épuisement émotionnel (EE-I et EE-F) sont élevées. Est-ce que les variables personnelles introduites comme variables de contrôle changent cette relation? Les tableaux 6 et 7 contiennent les résultats de ces analyses.

II s'en dégage que, autant pour les hommes que pour les femmes, I'accroissement de la pression du travail est en relation avec un accroissement de la fréquence et de l'intensité de l'épuisement émotionnel (EE-I et EE-F). Pour ce qui est de la santé, la pression du travail n'est pas en relation avec l'épuisement émotionnel lorsque les intervenants se déclarent en excellente santé. Mais à partir du moment où l'état de santé déclaré est un peu moins bon ou passable, on constate une influence très forte de la pression du travail sur les deux aspects de l'épuisement émotionnel (EE-I et EE-F).

\section{TABLEAU 6}

Influence des variables de contrôle sur la relation entre la pression du travail et l'intensité de l'épuisement émotionnel

\begin{tabular}{|c|c|c|c|c|c|c|}
\hline $\begin{array}{l}\text { Aspect cadre } \\
\text { de travail }\end{array}$ & $\begin{array}{r}\text { Variables } \\
\text { de contrôle }\end{array}$ & $\begin{array}{r}\text { Sous- } \\
\text { groupes }\end{array}$ & $N$ & $\begin{array}{l}\text { Coef. } \\
\text { de corr. }\end{array}$ & $\begin{array}{r}\text { Valeur } \\
\text { du } T\end{array}$ & $\begin{array}{r}\text { Sign. } \\
\text { du coef. }\end{array}$ \\
\hline \multirow{4}{*}{$\begin{array}{l}\text { Pression } \\
\text { du travail }\end{array}$} & Sexe & $\begin{array}{r}\text { Homme } \\
\text { Femme }\end{array}$ & $\begin{array}{l}35 \\
60\end{array}$ & $\begin{array}{l}0,334 \\
0,354\end{array}$ & $\begin{array}{l}2,154 \\
2,885\end{array}$ & $\begin{array}{l}0,05^{*} \\
0,006^{*}\end{array}$ \\
\hline & Santé & $\begin{array}{r}\text { Excellente } \\
\text { Bonne } \\
\text { Passable }\end{array}$ & $\begin{array}{r}25 \\
62 \\
8\end{array}$ & $\begin{array}{l}0,031 \\
0,441 \\
0,789\end{array}$ & $\begin{array}{l}0,151 \\
3,807 \\
3,142\end{array}$ & $\begin{array}{l}0,876 \\
0,001^{*} \\
0,020^{*}\end{array}$ \\
\hline & $\begin{array}{r}\text { Situation } \\
\text { de vie }\end{array}$ & $\begin{array}{r}\text { Célibataire } \\
\text { Marié }\end{array}$ & $\begin{array}{l}32 \\
63\end{array}$ & $\begin{array}{l}0,229 \\
0,478\end{array}$ & $\begin{array}{l}1,289 \\
4,250\end{array}$ & $\begin{array}{l}0,205 \\
0,000^{*}\end{array}$ \\
\hline & $\begin{array}{r}\text { Autre } \\
\text { formation }\end{array}$ & $\begin{array}{l}\text { Oui } \\
\text { Non }\end{array}$ & $\begin{array}{l}18 \\
77\end{array}$ & $\begin{array}{l}0,286 \\
0,393\end{array}$ & $\begin{array}{l}1,195 \\
3,701\end{array}$ & $\begin{array}{l}0,248 \\
0,001^{*}\end{array}$ \\
\hline
\end{tabular}




\section{TABLEAU 7}

Influence des variables de contrôle sur la relation entre la pression du travail et la fréquence de l'épuisement émotionnel

\begin{tabular}{|c|c|c|c|c|c|c|}
\hline $\begin{array}{l}\text { Aspect cadre } \\
\text { de travail }\end{array}$ & $\begin{array}{r}\text { Variables } \\
\text { de contrôle }\end{array}$ & $\begin{array}{r}\text { Sous- } \\
\text { groupes }\end{array}$ & $N$ & $\begin{array}{r}\text { Coef. } \\
\text { de corr. }\end{array}$ & $\begin{array}{r}\text { Valeur } \\
\text { du } T\end{array}$ & $\begin{array}{r}\text { Sign. } \\
\text { du coef. }\end{array}$ \\
\hline \multirow{5}{*}{$\begin{array}{l}\text { Pression } \\
\text { du travail }\end{array}$} & Sexe & $\begin{array}{r}\text { Homme } \\
\text { Femme }\end{array}$ & $\begin{array}{l}35 \\
60\end{array}$ & $\begin{array}{l}0,580 \\
0,416\end{array}$ & $\begin{array}{l}4,089 \\
3,482\end{array}$ & $\begin{array}{l}0,000^{*} \\
0,001^{*}\end{array}$ \\
\hline & Santé & $\begin{array}{r}\text { Excellente } \\
\text { Bonne } \\
\text { Passable }\end{array}$ & $\begin{array}{r}25 \\
62 \\
8\end{array}$ & $\begin{array}{l}0,194 \\
0,523 \\
0,870\end{array}$ & $\begin{array}{l}0,946 \\
4,755 \\
4,331\end{array}$ & $\begin{array}{l}0,644 \\
0,000^{*} \\
0,005^{*}\end{array}$ \\
\hline & $\begin{array}{r}\text { Situation } \\
\text { de vie }\end{array}$ & $\begin{array}{r}\text { Célibataire } \\
\text { Marié }\end{array}$ & $\begin{array}{l}32 \\
63\end{array}$ & $\begin{array}{l}0,388 \\
0,566\end{array}$ & $\begin{array}{l}2,306 \\
5,536\end{array}$ & $\begin{array}{l}0,027^{*} \\
0,000^{*}\end{array}$ \\
\hline & $\begin{array}{l}\text { Nombre d'int. } \\
\text { dans le milieu }\end{array}$ & $\begin{array}{r}3-10 \\
11-16\end{array}$ & $\begin{array}{l}36 \\
59\end{array}$ & $\begin{array}{l}0,095 \\
0,628\end{array}$ & $\begin{array}{l}0,554 \\
6,064\end{array}$ & $\begin{array}{l}0,590 \\
0,000^{*}\end{array}$ \\
\hline & $\begin{array}{r}\text { Domaine } \\
\text { de pratique }\end{array}$ & $\begin{array}{l}\text { Autorité } \\
\text { Volont. } \\
\text { R. + M.D. } \\
\text { Org. com. }\end{array}$ & $\begin{array}{r}34 \\
42 \\
11 \\
8\end{array}$ & $\begin{array}{l}0,507 \\
0,321 \\
0,534 \\
0,713\end{array}$ & $\begin{array}{l}3,326 \\
2,143 \\
1,904 \\
2,490\end{array}$ & $\begin{array}{l}0,003^{*} \\
0,036^{*} \\
0,087 \\
0,046^{*}\end{array}$ \\
\hline
\end{tabular}

$* p \leq 0,05$

Lorsque les intervenants sont mariés ou vivent en couple, I'influence d'une pression du travail plus forte accentue l'intensité de l'épuisement émotionnel (EE-I), mais quand I'accroissement de cette pression se fait sentir sur la fréquence de l'épuisement émotionnel (EE-F), cette relation est présente autant pour les intervenants célibataires que pour les intervenants mariés ou vivant en couple.

Le fait de ne pas avoir participé à d'autres formations en cours d'emploi est lui aussi associé à une intensité plus grande d'épuisement émotionnel (EE-I), alors qu'aucune constatation du genre n'existe pour la fréquence de l'épuisement émotionnel (EE-F). Par ailleurs, il y a une relation significative $(p \leq 0,05)$ entre l'augmentation de la pression du travail et l'accroissement de la fréquence de l'épuisement émotionnel (EE-F) dans les milieux où il y a plus de dix intervenants, relation qu'on ne retrouve pas dans les milieux plus petits. La relation entre la pression du travail et la fréquence de l'épuisement émotionnel (EE-F) se retrouve dans la plupart des domaines de pratique, sauf dans celui des ressources (CSS) et celui du maintien à domicile (CLSC).

Étant donné que le lien entre la clarté et l'intensité de l'épuisement émotionnel (EE-I) est faible $(r=-0,21)$, nous $n$ 'introduisons pas ici de variables de contrôle dans cette relation. Par ailleurs, il en va autrement pour ce 
qui est de la relation entre la clarté et la fréquence de l'épuisement émotionnel (EE-F) où le coefficient de corrélation est de $-0,52$. On remarque $d^{\prime}$ abord au tableau 8 que la santé et la situation de vie n'ont pas d'influence sur cette relation. Toutefois, il appert que la relation est présente pour les femmes et ne l'est pas pour les hommes. C'est donc dire que, de façon significative $(p \leq 0,05)$, les femmes vivent plus souvent que les hommes le sentiment d'être épuisées émotionnellement dans les situations où les directives sont moins claires.

Il y a aussi une relation différente entre la clarté et la fréquence de I'épuisement émotionnel (EE-F) pour les intervenants issus de milieux de travail plus petits: une moins grande clarté des directives s'accompagne d'une fréquence plus forte de l'épuisement émotionnel dans les milieux avec dix employés et moins, alors qu'il n'y a pas de différence chez les répondants dans les milieux qui comptent de onze à seize intervenants.

Enfin, on fait également la même constatation en contexte d'autorité: les intervenants y vivent plus souvent le sentiment d'être épuisés émotionnellement lorsque la clarté des directives venant de leurs supérieurs immédiats diminue. Cette relation n'est pas significative dans les autres domaines de pratique.

\section{Tableau 8}

Influence des variables de contrôle sur la relation entre la clarté et la fréquence de l'épuisement émotionnel

\begin{tabular}{|c|c|c|c|c|c|c|}
\hline $\begin{array}{l}\text { Aspect cadr } \\
\text { de travail }\end{array}$ & $\begin{array}{r}\text { Variables } \\
\text { de contrôle }\end{array}$ & $\begin{array}{r}\text { Sous- } \\
\text { groupes }\end{array}$ & $N$ & $\begin{array}{r}\text { Coef. } \\
\text { de corr. }\end{array}$ & $\begin{array}{r}\text { Valeur } \\
\text { du T }\end{array}$ & $\begin{array}{r}\text { Sign. } \\
\text { du coef. }\end{array}$ \\
\hline \multirow{5}{*}{$\begin{array}{l}\text { Pression } \\
\text { du travail }\end{array}$} & Sexe & $\begin{array}{l}\text { Homme } \\
\text { Femme }\end{array}$ & $\begin{array}{l}35 \\
60\end{array}$ & $\begin{array}{l}-0,031 \\
-0,372\end{array}$ & $\begin{array}{l}0,177 \\
3,053\end{array}$ & $\begin{array}{l}0,855 \\
0,004^{*}\end{array}$ \\
\hline & Santé & $\begin{array}{r}\text { Excellente } \\
\text { Bonne } \\
\text { Passable }\end{array}$ & $\begin{array}{r}25 \\
62 \\
8\end{array}$ & $\begin{array}{l}-0,165 \\
-0,179 \\
-0,408\end{array}$ & $\begin{array}{l}0,802 \\
1,405 \\
1,044\end{array}$ & $\begin{array}{l}0,564 \\
0,162 \\
0,317\end{array}$ \\
\hline & $\begin{array}{r}\text { Situation } \\
\text { de vie }\end{array}$ & $\begin{array}{r}\text { Célibataire } \\
\text { Marié }\end{array}$ & $\begin{array}{l}32 \\
63\end{array}$ & $\begin{array}{l}-0,267 \\
-0,191\end{array}$ & $\begin{array}{l}1,520 \\
1,520\end{array}$ & $\begin{array}{l}, 135 \\
0,130\end{array}$ \\
\hline & $\begin{array}{l}\text { Nombre d'int. } \\
\text { dans le milieu }\end{array}$ & $\begin{array}{r}3-10 \\
11-16\end{array}$ & $\begin{array}{l}36 \\
59\end{array}$ & $\begin{array}{l}-0,354 \\
-0,161\end{array}$ & $\begin{array}{l}2,206 \\
1,235\end{array}$ & $\begin{array}{l}0,032^{*} \\
0,220\end{array}$ \\
\hline & $\begin{array}{r}\text { Domaine } \\
\text { de pratique }\end{array}$ & $\begin{array}{l}\text { Autorité } \\
\text { Volont. } \\
\text { R. + M.D. } \\
\text { Org. com. }\end{array}$ & $\begin{array}{r}34 \\
42 \\
11 \\
8\end{array}$ & $\begin{array}{l}-0,366 \\
-0,208 \\
-0,289 \\
-0,045\end{array}$ & $\begin{array}{l}2,224 \\
1,344 \\
0,907 \\
0,110\end{array}$ & $\begin{array}{l}0,031^{*} \\
0,184 \\
0,609 \\
0,912\end{array}$ \\
\hline
\end{tabular}




\section{CONCLUSION}

Malgré quelques limites particulières attribuables au fait qu'on ne connaît pas complètement la validité de l'EET en milieu québécois, que le taux de participation a été plutôt faible dans trois CLSC (moins de 50\%) et qu'une seule région très particulière du Québec soit couverte, il n'en reste pas moins que les constatations faites permettent de jeter un éclairage unique sur ce problème de l'épuisement professionnel pour ensuite en utiliser les constatations dans la pratique de la gestion. Mais il est important de garder à l'esprit que la question de l'épuisement professionnel ne peut être abordée sans qu'un certain nombre de considérations soient mises en relief. C'est une réalité complexe et multidimensionnelle que nous avons étudiée.

Comme on l'a vu dans cette étude, il faut tenir compte de plusieurs catégories de facteurs qui paraissent lui être reliées. On doit aussi garder en mémoire, comme Courage et Williams (1987) le font ressortir, que le problème relève d'une interaction entre le personnel, l'organisation et la clientèle. Ces conditions forcent l'observateur à reconnaître la complexité du phénomène et la nécessité qui en découle de l'aborder dans I'action en utilisant une approche globale qui tienne compte du travailleur en interaction avec son environnement.

D'ailleurs, à titre d'exemple, soulignons que le Centre de services sociaux de la Gaspésie et des îles a profité de cette recherche pour entreprendre une démarche de réflexion et d'action en vue d'améliorer le climat de travail dans l'établissement et, par conséquent, de diminuer les risques d'épuisement professionnel. Les résultats propres aux six points de service que comptait alors le CSS ont été transmis à la direction et aux intervenants. Ces résultats faisaient état du climat de travail, du degré d'épuisement et des liens que cette recherche dégage entre les deux. D'une part, il était possible de comparer chaque point de service aux autres et à l'ensemble de l'établissement. D'autre part, il devenait pensable d'agir sur le milieu, puisque des caractéristiques positives et négatives étaient clairement décrites. Finalement, en faisant ressortir celles d'entre elles qui avaient une influence sur le degré d'épuisement aux six sous-échelles de Maslach, il était possible de définir des cibles de changement.

D'une façon volontaire, les intervenants de chaque point de service ont eu la possibilité de s'associer à leurs supérieurs immédiats pour discuter des conclusions de l'étude qui s'appliquaient à leur milieu et penser à des façons d'améliorer le climat de travail. Dans un point de service, par exemple, on avait constaté que la pression du travail s'avérait très forte et la clarté des directives très faible. En ce qui concerne les degrés d'épuisement, les deux sous-échelles de l'épuisement émotionnel présentaient des résultats élevés. Étant donné que l'étude dégage une relation significative entre la pression du travail et la clarté, d'une part, et les deux sous-échelles de l'épuisement 
émotionnel, d'autre part, il devenait évident que la charge de travail très grande et le manque de clarté des directives favorisaient la fréquence et l'intensité du sentiment que vivaient les employés d'être vidés émotionnellement.

Pour ce qui est des variables personnelles, elles ont été utiles pour préciser l'impact des liens entre le cadre de travail et l'épuisement. Ainsi, dans le dernier exemple du point de service, en sachant que le lien entre la pression du travail et l'intensité de l'épuisement émotionnel s'applique surtout aux gens mariés ou vivant en couple, il a été possible de déterminer des cibles de changement plus précises à partir d'observations plus «pointues». L'utilisation d'informations du même genre a aussi été possible à la suite de la constatation des différences existant dans l'épuisement des employés qui n'ont pas suivi de formation en cours d'emploi par rapport à ceux qui en ont suivi.

En somme, même si ce n'était pas là l'objectif de départ de la recherche, I'application qui a été faite de ses conclusions démontre qu'une meilleure compréhension du phénomène et des facteurs qui favorisent son apparition peuvent entraîner des actions appropriées pour le prévenir.

\section{Références bibliographiques}

BERNIER, Diane (1989a). «Le burnout chez les travailleurs sociaux: omniprésent, mais mal connu », Intervention, $\mathrm{n}^{\circ} 82$ : 13-21.

BERNIER, Diane (1989b et 1992). Étude longitudinale des cas d'épuisement professionnel grave chez les intervenants sociaux des CSS du Québec. Document de travail déposé à la table des DSP, ACSSQ ; 7-8 décembre 1989. (Rapport final 1992)

CherNisS, C. (1980a). Staff Burn Out. Beverly Hills: Sage Publications inc.

CHERNISS, C. (1980b). Professionnal Burnout in Human Service Organizations. New York: Praeger Publishers.

CONSTABLE, J. et D.W. RuSSELl (1986). «The Effect of Social Support and the Work Environment Upon Burnout Among Nurses», The Journal of Human Stress, vol. 12, no $1: 20-26$.

COURAGE, M.M. et D.D. Williams (1987) «An approach to the study of burnout in professional care providers in human service organizations », Journal of Social Service Research, vol. 10, no 1: 7-22.

DUQUETTE, A., S. KÉROUAC et L. BEAUDET (1990). Facteurs reliés à l'épuisement professionnel du personnel infirmier: un examen critique d'études empiriques. Rapport présenté au Conseil québécois de la recherche sociale, Université de Montréal.

FIMIAN, M.J. (1984). «Organizational Variables Related to Stress and Burnout in Community-Based Programs », Education and Training of the Mentally Retarded, vol. 19, n $3:$ 201-209.

FIRTH, H., J. MCINTEE, P. MCKEOWN et P. BRITTON (1986). «Burnout and professional depression related concepts », Journal of Advanced Nursing, vol. 11, $\mathrm{n}^{\circ}$ 6: 633-641. 
FONG, C.M. (1984). A study of the relationship between role and overload, social support and burnout among nursing educators. Unpublished Doctoral Dissertation. University of California, dans A. DUQUETTE, S. KÉROUAC et L. BEAUDET (1990). Facteurs reliés à l'épuisement professionnel du personnel infirmier: un examen critique d'études empiriques. Rapport présenté au Conseil québécois de la recherche sociale, Université de Montréal.

GeVRY, M. et M. AlAIN (1988). «L'épuisement professionnel dans le secteur des services humains », Intervention, $\mathrm{n}^{\circ} 80: 60-65$.

GOlEMBIEWSKI, R.T., R. HILlES et R. DAly (1987). "Some Effects of Multiple O.D. Interventions on Burnout and WorkSite Features », The Journal of Applied Behavioral Science, vol. 23, n $3:$ 295-313.

MASLACH, C. et S.E. JACKSON (1981). The Maslach Burnout Inventory. Palo Alto: Consulting Psychologists Press, Inc.

MASLACH, C. et S.E. JACKSON (1985). "The Role of Sex and Family Variables in Burnout», Sex Roles, vol. 12, n 7-8: 837-851.

MCCULlOCH, A. et L. O'BRIEN (1986). «The Organizational Determinants of Worker Burnout», Children and Youth Services Review, 8: 175-190.

MEUNIER, C. (1984). "Êtes-vous sur la voie du burn out? », Affaires, septembre 1984 : 23-26.

MoOS, R.H. (1986).Work Environment Scale Manual. Second Edition. Palo Alto: Consulting Psychologists Press, Inc.

PINES, A., E. ARONSON et D. KAFry (1982). "Burnout»: Se vider dans la vie et au travail. Collection Actualisation, Montréal : Éd. Le Jour.

Rosenthal, D., M. TeAgue, P. Retish, J. West et R. WeSSel (1983). «The Relationship Between Work Environment Attributes and Burnout », Journal of Leisure Research, vol. 15, $\mathrm{n}^{\circ} 2$ : 125-135.

SELYE, H. (1975). Le stress de la vie. Ottawa: Gallimard/Lacombe.

SYNDICAT DES PROFESSIONNELS DU GOUVERNEMENT DU QUÉBEC (1986). Le Stress au boulot. Brochure du comité d'information, avril 1986.

VILLENEUVE, J.-P. (1991). Le cadre de travail et le degré d'épuisement chez les intervenants sociaux en Gaspésie. Mémoire de maîtrise, Université Laval, Québec, École des gradués.

WADE, D.C., E. COOlEY et V. SAVICK (1986). "A Longitudinal Study of Burnout», Children and Youth Services Review, 8: 161-173. 